\title{
Resistin expression in adipose tissues and its effect on glucose metabolism in rats with brain injury
}

\author{
R. Zhang', Z.Y. Wang', L.L. Zhu', F. Wu', D.Q. Chen ${ }^{1}$, L.F. Sun ${ }^{1}$ and Z.Q. Lu ${ }^{2}$ \\ ${ }^{1}$ Department of Emergency, \\ The Second Affiliated Hospital of Wenzhou Medical University, Wenzhou, China \\ ${ }^{2}$ Department of Emergency, First Affiliated Hospital of Wenzhou Medical University, \\ Wenzhou, China \\ Corresponding author: D.Q. Chen \\ E-mail: chendaqing_|@163.com \\ Genet. Mol. Res. 15 (1): gmr.15017659 \\ Received September 17, 2015 \\ Accepted December 9, 2015 \\ Published February 12, 2016 \\ DOI http://dx.doi.org/10.4238/gmr.15017659
}

ABSTRACT. Resistin (RSTN) expression in subcutaneous adipose tissue, and its effect on glucose metabolism in rats with traumatic brain injury, was investigated using real-time PCR, western blots, and enzyme linked immunoassays. Our results show that the expression of RSTN mRNA $(3.192 \pm 0.046,4.016 \pm 0.010,6.004 \pm 0.020,8.213 \pm 0.013,11.199 \pm$ $0.174,15.094 \pm 0.030)$, protein levels $(1.79 \pm 0.05,1.98 \pm 0.07,2.75 \pm$ $0.08,3.19 \pm 0.08,4.25 \pm 0.11,4.48 \pm 0.07)$, levels of serum insulin $(512.96$ $\pm 1.21,580.57 \pm 1.52,769.71 \pm 2.22,826.08 \pm 2.03,1262.25 \pm 3.40$, $1512.80 \pm 3.93)$, and fasting blood glucose levels (10.277 $\pm 0.040,12.776$ $\pm 0.038,13.403 \pm 0.263,14.698 \pm 0.100,16.637 \pm 0.110,19.416 \pm 0.025)$ were significantly higher in the traumatic rat group compared to the control group $(P<0.05)$. Quantitative insulin sensitivity check index (QUICKI) was significantly lower in the traumatic group $(-8.570 \pm 0.005,-8.912 \pm 0.004$, $-9.241 \pm 0.022,-9.404 \pm 0.007,-9.952 \pm 0.007,-10.288 \pm 0.002)$ than in the control group $(-7.633 \pm 0.003,-7.639 \pm 0.004,-7.637 \pm 0.006,-7.643$ $\pm 0.003,-7.636 \pm 0.006,-7.634 \pm 0.004)(P<0.05)$. Single factor linear 
correlation analysis showed that there was a significant negative correlation between RSTN expression and QUICKI $(-0.983, P<0.05)$ in the traumatic group. The increase in RSTN expression in the subcutaneous adipose tissue of rats with traumatic brain injury is likely related to the indexes of glycometabolism, including serum insulin, fasting blood glucose, and QUICKI. Our results lead us to conclude that RSTN may play an important role in the process of insulin resistance in rats with traumatic brain injury.

Key words: Traumatic brain injury; Resistin; Insulin resistance; Fasting blood glucose

\section{INTRODUCTION}

Traumatic brain injury (TBI) is the primary type of injury correlated with death and permanent disability. The disease mechanisms of TBI and secondary brain injury are complex. Recently, the hormone resistin (RSTN) was found in to play a role in the pathophysiology of diabetes (Wiesner et al., 2006). At present, studies on RSTN mainly focus on its relationships with obesity, insulin resistance, and diabetes. Many studies considered that RSTN was the key factor affecting insulin resistance (Reilly et al., 2005; Qiao and Tang, 2010). Some studies suggest that RSTN, being the intermediary link between obesity and insulin resistance, was positively correlated with insulin resistance, physiological effects of insulin resistance, increased blood glucose, and inhibition of adipocyte differentiation (Kaser et al., 2003; Kawanami et al., 2004). Steppan et al. (2001) postulated that the RSTN was the bridge between obesity, insulin resistance, and type-2 diabetes. There are few reports on the relationship between RSTN and traumatic insulin resistance. The only indication was from a study on the incidence and development of traumatic insulin resistance, which showed that metabolic disturbance was the common link between RSTN and traumatic insulin resistance in traumatic brain injury. In this study, we investigated the expression of RSTN in subcutaneous adipose tissue of rats with traumatic brain injury, at the gene and protein levels, and further clarified the effects of RSTN on insulin resistance in rats with traumatic brain injury. The results provide new intervention targets and a theoretical basis for clinical treatment of traumatic brain injury.

\section{MATERIAL AND METHODS}

\section{Materials}

\section{Regents and instruments}

The anti-RSTN antibody (sc-16116) was purchased from Santa Cruz Biotechnologies (Santa Cruz, CA, USA) and GAPDH antibody (AB-P-R001) was purchased from Han Zhou Xian Zhi Biotechnology (Han Zhou Xian Zhi Biotechnology, China). The anti-goat rabbit IgG HPR secondary antibody (BA1060) was purchased from Wuhan Boster Biological Technology (Wuhan Boster Biological Technology, Wuhan, China). Rat serum RSTN ELISA detection kit was purchased from Shanghai Yi Li Biotech (Shanghai Yi Li Biotech, Shanghai, China). TRIZOL was purchased from Invitrogen (Invitrogen, Carlsbad, CA, USA). Reverse transcriptase was purchased from Fermentas (Fermentas, Burlington, Canada) and the PCR Kit was purchased from TaKaRa Biotechnology Co., Ltd. (Dalian, China). 


\section{Animals}

The 56 specific pathogen free, Sprague-Dawley (SD) male rats weighing between 300 to $350 \mathrm{~g}$ were provided by the Hubei Province Experimental Animal Study Centre.

\section{Methods}

\section{Groups and Methods}

The 56 SD rats were divided into a control group (8 rats) and a traumatic group (48 rats) randomly. The rats in the control group were treated only by opening the scalp and skull, while fluid percussion was used to induce traumatic brain injury in rats of the traumatic group (Li et al., 1999) with a shocking pressure of 300 kPa. After 12 h, 24 h, 72 h, 1 week, 2 weeks, and 4 weeks, blood was collected from the caudal vessel. The subcutaneous adipose tissue was obtained after sacrificing the rats, and then stored at $-70^{\circ} \mathrm{C}$. All treatments were conducted according to the guidelines established by the Canadian Council on Animal Care.

\section{Determination of expression level of the RSTN gene in subcutaneous fat by real-time PCR}

Total RNA from subcutaneous adipose tissue was extracted using Trizol, and the RNA concentration was determined by spectrophotometer at OD $260 \mathrm{~nm}$ and OD $280 \mathrm{~nm}$. The RNA concentration was obtained after conversion (1 OD $260 \mathrm{~nm}=40 \mu \mathrm{g}$ RNA). The RNA was reverse transcribed into cDNA. Real time PCRs were performed on an ABI 7900 System (Applied Biosciences, USA). The primer 5.0 software was used to design the following primers; $\beta$-actin Forward (F) primer: 5'-CACGATGGAGGGGCCGGACTCATC-3', $\beta$-actin Reverse (R) primer: 5'-TAAAGACCTCTATGCCAACACAGT-3'; RSTN F: 5'-CCAGAAGGCACAACCGTCACTA-3', RSTN R: 5'-TCAACCGTCCTCAGGAACCAA-3'. The cycling parameters were as follows: $50^{\circ} \mathrm{C}$ for $2 \mathrm{~min}, 1 \mathrm{cycle}$ at $95^{\circ} \mathrm{C}$ for $10 \mathrm{~min}$, then 40 cycles, at $60^{\circ} \mathrm{C}$ for $30 \mathrm{~s}$. The standard curve was estimated and used to determine the expression levels of RSTN and $\beta$-actin in rat subcutaneous adipose tissue. The ratio between RSTN mRNA and $\beta$-actin mRNA was used as the index to evaluate the expression level of RSTN mRNA.

\section{Determination of the expression of RSTN protein in subcutaneous fat by western blots}

Total protein of cells were extracted and detected according to the manufacturer protocol included in the kit. After protein denaturation and gel electrophoresis, the proteins were transferred onto membranes and blocked with $5 \%$ non-fatty milk blocking buffer for $2 \mathrm{~h}$, then incubated with RSTN antibody $(1: 500)$ and GAPDH antibody $(1: 1000)$ at $4^{\circ} \mathrm{C}$, overnight. The membranes were then washed 3 times with TBST and then incubated with anti-goat rabbit IgG HPR secondary antibody $(1: 20,000)$ at room temperature, and washed 3 times with TBST for $10 \mathrm{~min}$. The blots were exposed using enhanced chemiluminescence $(E C L)$. 


\section{Analysis of fasting blood glucose (FBG), serum insulin and insulin sensitivity index (ISI)}

ELISA was used to detect the FBG and serum insulin in each group according to the manufacturer protocol. The ISI was calculated according to the method described by $\mathrm{Li}$ et al. (1993) and reported as follows: ISI = 1 / (FPG $\times$ FINS), where FPG is the fasting plasma glucose, and FINS is the fasting insulin concentration. Since the data was not normally distributed, the ISI was transformed by natural logarithm, as insulin action index (IAI): IAI = -In (FPG $\times$ FINS). Insulin sensitivity and quantitative insulin sensitivity check index (QUICKI) at each time point was calculated in each group, separately.

\section{Statistical analysis}

Data analyses were performed using the SPSS13.0 software, and the results are reported as means \pm standard deviations. A one-way ANOVA was used to compare differences between groups, and correlation analysis was performed using single factor covariance analysis. A value of $\mathrm{P}<0.05$ was considered as statistically significant.

\section{RESULTS}

\section{Expression of the RSTN gene in subcutaneous fat}

Compared to the control group, the gene expression of RSTN significantly increased in subcutaneous fat in the traumatic group at each time point $(12 \mathrm{~h}, 24 \mathrm{~h}, 72 \mathrm{~h}, 1$ week, 2 weeks, and 4 weeks, $P<0.05)$. In the traumatic group, the expression of RSTN increased as the time increased $(P<0.05$, Table 1).

Table 1. Expression of the RSTN gene in subcutaneous fat of each group (means $\pm S D, N=8$ ).

\begin{tabular}{|c|c|c|c|c|c|c|}
\hline \multirow{2}{*}{ Group } & \multicolumn{6}{|c|}{ Time } \\
\hline & $12 \mathrm{~h}$ & $24 \mathrm{~h}$ & $72 \mathrm{~h}$ & 1 week & 2 weeks & 4 weeks \\
\hline $\begin{array}{l}\text { Control group } \\
\text { Injury group }\end{array}$ & $\begin{array}{c}1.115 \pm 0.006 \\
3.192 \pm 0.046^{*}\end{array}$ & $\begin{array}{c}1.143 \pm 0.012 \\
4.016 \pm 0.010^{*}\end{array}$ & $\begin{array}{l}1.216 \pm 0.132 \\
6.004 \pm 0.020^{*}\end{array}$ & $\begin{array}{l}1.144 \pm 0.014 \\
8.213 \pm 0.013^{*}\end{array}$ & $\begin{array}{l}1.142 \pm 0.034 \\
11.199 \pm 0.174^{*}\end{array}$ & $\begin{array}{l}1.124 \pm 0.002 \\
15.094 \pm 0.030^{*}\end{array}$ \\
\hline $\begin{array}{l}T \text { value } \\
P \text { value }\end{array}$ & $\begin{array}{c}12,184.490 \\
0.000\end{array}$ & $\begin{array}{c}20,6757.082 \\
0.000\end{array}$ & $\begin{array}{c}76,76.268 \\
0.000\end{array}$ & $\begin{array}{c}81,7171.016 \\
0.000\end{array}$ & $\begin{array}{c}19,275.986 \\
0.000\end{array}$ & $\begin{array}{c}1,259,340.777 \\
0.000\end{array}$ \\
\hline
\end{tabular}

${ }^{*}$ Compared with the control group, $\mathrm{P}<0.05$.

\section{Protein expression of RSTN in subcutaneous fat}

Compared to the control group, the expression of RSTN protein significantly increased in the traumatic group at each time point $(12 \mathrm{~h}, 24 \mathrm{~h}, 72 \mathrm{~h}, 1$ week, 2 weeks, and 4 weeks, $\mathrm{P}<0.05)$. In the traumatic group, the expression of RSTN increased as time increased $(P<0.05$, Table 2$)$. 
Table 2. Expression of resistin in subcutaneous fat of each group (means $\pm S D, N=8$ ).

\begin{tabular}{l|c|c|c|c|c|c}
\hline \multirow{2}{*}{ Group } & \multicolumn{7}{|c}{ Time } \\
\cline { 2 - 7 } & $12 \mathrm{~h}$ & $24 \mathrm{~h}$ & $72 \mathrm{~h}$ & 1 week & 2 weeks & 4 weeks \\
\hline Control group & $1.01 \pm 0.04$ & $1.01 \pm 0.02$ & $1.09 \pm 0.05$ & $1.03 \pm 0.04$ & $1.02 \pm 0.03$ & $1.01 \pm 0.03$ \\
Injury group & $1.79 \pm 0.05^{\star}$ & $1.98 \pm 0.07^{\star}$ & $2.75 \pm 0.08^{*}$ & $3.19 \pm 0.08^{\star}$ & $4.25 \pm 0.11^{*}$ & $4.48 \pm 0.07^{*}$ \\
\hline F value & 858.245 & $1,029.852$ & $1,782.017$ & $3,137.190$ & $5,346.208$ & $13,227.126$ \\
P value & 0.000 & 0.000 & 0.000 & 0.000 & 0.000 & 0.000 \\
\hline
\end{tabular}

*Compared with the control group, $\mathrm{P}<0.05$.

\section{Changes in blood glucose, insulin, and insulin sensitivity index}

Compared to the control, the blood glucose, and serum insulin levels significantly increased in the traumatic group, reaching a maximum at 4 weeks, with significant differences $(P<0.05$, Tables 3 and 4). In addition, the QUICKI significantly decreased in the traumatic group compared to the control group, and this difference was statistically significant $(P<0.05$, Table 5$)$.

\begin{tabular}{|c|c|c|c|c|c|c|}
\hline \multirow{2}{*}{ Group } & \multicolumn{6}{|c|}{ Time } \\
\hline & $12 \mathrm{~h}$ & $24 \mathrm{~h}$ & $72 \mathrm{~h}$ & 1 week & 2 weeks & 4 weeks \\
\hline \multirow{2}{*}{$\begin{array}{l}\text { Control group } \\
\text { Injury group }\end{array}$} & $5.838 \pm 0.006$ & $5.645 \pm 0.007$ & $5.642 \pm 0.018$ & $5.630 \pm 0.008$ & $5.627 \pm 0.012$ & $5.625 \pm 0.004$ \\
\hline & $10.277 \pm 0.040^{*}$ & $12.776 \pm 0.038^{*}$ & $13.403 \pm 0.263^{*}$ & $14.698 \pm 0.100^{*}$ & $16.637 \pm 0.110^{*}$ & $19.416 \pm 0.025^{*}$ \\
\hline F value & $79,006.321$ & $202,905.051$ & $5,214.756$ & $49,253.847$ & $59,895.613$ & $1,763,452.088$ \\
\hline$P$ value & 0.000 & 0.000 & 0.000 & 0.000 & 0.000 & 0.000 \\
\hline
\end{tabular}

${ }^{*}$ Compared with the control group, $\mathrm{P}<0.05$.

Table 4. Blood insulin levels of rats in each group (means $\pm S D, N=8, p g / m L)$.

\begin{tabular}{l|c|c|c|c|c|c}
\hline \multirow{2}{*}{ Group } & \multicolumn{5}{|c}{ Time } \\
\cline { 2 - 7 } & $12 \mathrm{~h}$ & $24 \mathrm{~h}$ & $72 \mathrm{~h}$ & 1 week & 2 weeks & 4 weeks \\
\hline Control group & $366.28 \pm 1.04$ & $367.57 \pm 2.16$ & $367.57 \pm 2.16$ & $370.30 \pm 0.98$ & $368.11 \pm 1.94$ & $367.60 \pm 1.44$ \\
Injury group & $512.96 \pm 1.21^{*}$ & $580.57 \pm 1.52^{*}$ & $769.71 \pm 2.22^{*}$ & $826.08 \pm 2.03^{*}$ & $1262.25 \pm 3.40^{*}$ & $1512.80 \pm 3.93^{*}$ \\
\hline F value & $50,733.605$ & $74,820.505$ & $101,034.264$ & $245,244.509$ & $2,398,461.950$ & $449,227.343$ \\
$P$ value & 0.000 & 0.000 & 0.000 & 0.000 & 0.000 & 0.000 \\
\hline
\end{tabular}

${ }^{*}$ Compared with the control group, $\mathrm{P}<0.05$.

Table 5. QUICKI ( $Q$ value) of rats in each group (means $\pm S D, N=8$ ).

\begin{tabular}{l|c|c|c|c|c|c}
\hline \multirow{2}{*}{ Group } & \multicolumn{5}{|c}{ Time } \\
\cline { 2 - 7 } & $12 \mathrm{~h}$ & $24 \mathrm{~h}$ & $72 \mathrm{~h}$ & 1 week & 2 weeks & 4 weeks \\
\hline Control group & $-7.633 \pm 0.003$ & $-7.639 \pm 0.004$ & $-7.637 \pm 0.006$ & $-7.643 \pm 0.003$ & $-7.636 \pm 0.006$ & $-7.634 \pm 0.004$ \\
Injury group & $-8.570 \pm 0.005^{*}$ & $-8.912 \pm 0.004^{*}$ & $-9.241 \pm 0.022^{*}$ & $-9.404 \pm 0.007^{*}$ & $-9.952 \pm 0.007^{*}$ & $-10.288 \pm 0.002^{*}$ \\
\hline F value & $143,348.774$ & $299,316.445$ & $30,380.647$ & $282,452.326$ & $361,983.697$ & $2,365,743.974$ \\
P value & 0.000 & 0.000 & 0.000 & 0.000 & 0.000 & 0.000 \\
\hline
\end{tabular}

${ }^{*}$ Compared with the control group, $\mathrm{P}<0.05$. 


\section{Relationship between the expression of RSTN gene in the traumatic group and QUICKI}

The ISI of the traumatic group is shown in Figure 1, where the $Q$ value is on the $\mathrm{X}$-axis, and expression of the RSTN gene is on the Y-axis.

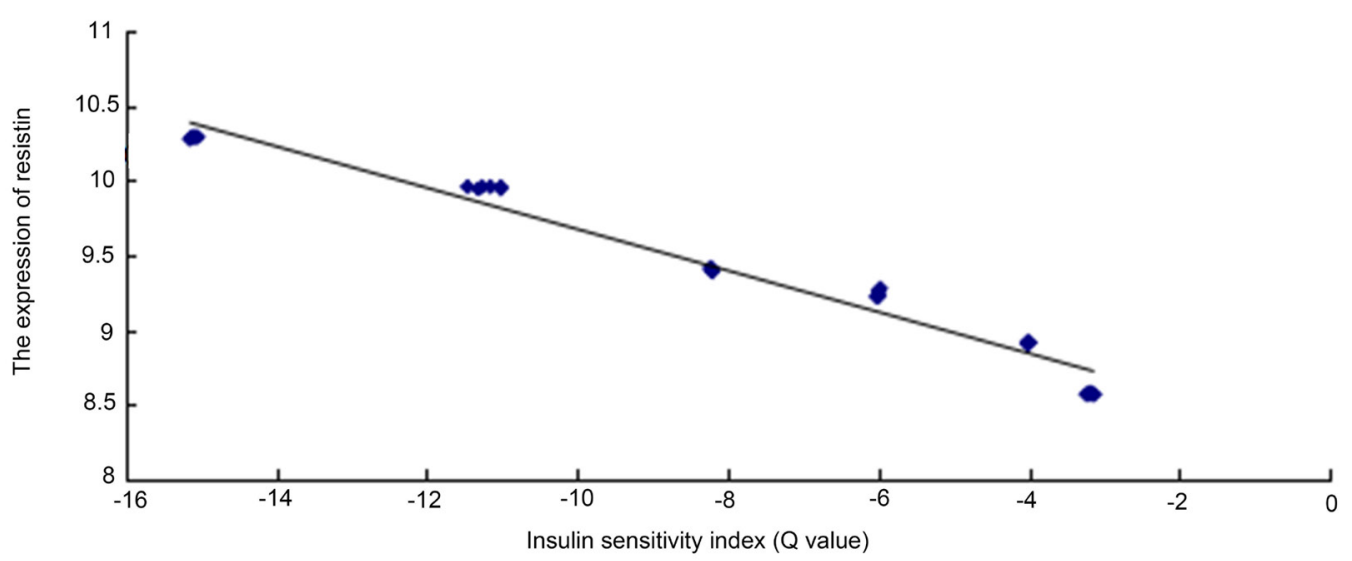

Figure 1. Relationship between the expression of resistin subcutaneous fat and insulin sensitivity index.

Overall, we found that RSTN and QUICKI showed a completely symmetric negative correlation. With further correlation analysis incorporating time / group, the results revealed that the expression of RSTN gene in fat tissues was significantly negatively correlated with insulin sensitivity, with a t correlation coefficients of $-0.983(P=0.000)$.

\section{DISCUSSION}

The RSTN multiplies a peptide hormone mainly secreted by the adipocytes. One study (Zhou et al., 2011) suggested that the serum RSTN concentration were positively correlated with body fat distribution, which revealed that the more the body fat, the higher the serum RSTN concentration. Accordingly, subcutaneous fat in rats was as the focus of this study, in order to exclude the influence of body fat. The previous study (Chen et al., 2010) showed that RSTN expression increased significantly in the brain tissue, including the hippocampus and the hypothalamus, of rats with traumatic brain injury, and the RSTN expression level increased according to the severity of the injury. However, no further investigation was conducted on the protein translation of RSTN. In this study, we investigated the expression of RSTN mRNA and protein levels in subcutaneous fat of rat with traumatic brain injury. The results showed that RSTN increased to different degrees compared to the control group after 4 weeks of injury, and the tendency was consistent, which proved the reliability of Chen et al. (2010).

The insulin resistance is sensitivity of body to insulin, and is common in metabolic diseases such as type-2 diabetes and abdominal adiposis. At the same time, it is also common in emergency situations, such as when the body undergoes an operation or suffers an injury, and is one of the key factors that influence body physiology and metabolism after injury and operations (Ljungqvist et al., 2000). At present, studies on emergency insulin resistance is mainly conducted 
in clinical trials in patients for selective operation, but there are few clinical and basic studies on emergency insulin resistance done under TBI. It is common that injuries (including traumatic brain injury) induce emergency high glucose reactions (emergency insulin resistance) (Ljungqvist et al., 2000; Tsatsoulis et al., 2013), that appear similar to the high glucose and insulin levels in type-2 diabetes. However, the disease mechanism is not completely understood. Steppan et al. (2001) found that in mice treated with recombined RSTN by intravenous injection, the blood glucose and serum insulin levels increased to different degrees, which suggested that glucose tolerance during injury was not caused by a secretion disorder of insulin, but is related to RSTN that weakened the effects of insulin. Hartman et al. (2002) found that inhibiting the expression of RSTN in mature adipose could decrease the blood glucose. Hartman et al. (2002) showed that the expression of RSTN is closely associated with glucose metabolism, which might participate in progression of insulin resistance by influencing the sensitivity of insulin. The results of previous study (Chen et al., 2010) showed that the expression of the RSTN gene had a positive association with peripheral blood glucose levels in brain tissues of rat with traumatic brain injury, such as in the hippocampus and the hypothalamus, which suggests that the improvement of blood glucose levels in rats with traumatic brain injury is related to the increased expression of RSTN. Another line of evidence was that the blood glucose and serum insulin levels significantly increased in rats with traumatic brain injury, and were positively correlated with the expression levels of RSTN in the abdominal adiposis. However, decreasing insulin sensitivity index was negatively correlated with RSTN expression. The higher expression of RSTN, lower insulin sensitivity, and stronger insulin resistance suggests that RSTN might play an important role in insulin resistance of rats with traumatic brain injury. In addition, a few patients with traumatic brain injury showed no significant clinical emergency associated with high glucose reaction; therefore, further studies are required to clarify whether any relationship exists between brain injury and high glucose levels in the abdominal adiposis contents.

In the present study, we investigated the changes in insulin sensitivity in rats with traumatic brain injury by establishing the traumatic brain injury model. The analyses of the relationship between insulin sensitivity and RSTN expression in subcutaneous adipose tissues of rats provided the mechanisms underlying TBI; however, new intervention targets, and a theoretical basis for the clinical treatment of traumatic brain injury require further studies.

\section{ACKNOWLEDGMENTS}

Research supported by the Wenzhou Technology Office (\#Y20130256), the Medical Rescue Plan of Major Disaster Plan of the Twelfth Five-year Plan, and the Key Disciplines in Colleges and Universities in Zhejiang Province.

\section{REFERENCES}

Chen DQ, Deng YB, Lin LY and Zhu LL (2010). Change of gene rstn expression in brain tissues after traumatic brain injury. Zhonghua Chuang Shangzazhi 9: 845-847.

Hartman HB, Hu X, Tyler KX, Dalal CK, et al. (2002). Mechanisms regulating adipocyte expression of resistin. J. Biol. Chem. 277: 19754-19761.http://dx.doi.org/10.1074/jbc.M201451200

Kaser S, Kaser A, Sandhofer A, Ebenbichler CF, et al. (2003). Resistin messenger-RNA expression is increased by proinflammatory cytokines in vitro. Biochem. Biophys. Res. Commun. 309: 286-290. http://dx.doi.org/10.1016/j.bbrc.2003.07.003

Kawanami D, Maemura K, Takeda N, Harada T, et al. (2004). Direct reciprocal effects of resistin and adiponectin on vascular endothelial cells: a new insight into adipocytokine-endothelial cell interactions. Biochem. Biophys. Res. Commun. 314: 415-419.http://dx.doi.org/10.1016/j.bbrc.2003.12.104 
Li GW, Pan XR, Lillioja S and Peter H (1993). A new index of detecting insulin sensitivity in populations. Zhonghua Neike Zazhi 12: 656-660.

Li LX, Wang TY and Zhong ZY (1999). Compared analysis of brain jury of rats induced by freely falling body and hydraulic pressure. Zhonghua Shiyan Waike Zazhi 16: 447-448.

Ljungqvist O, Nygren J and Thorell A (2000). Insulin resistance and elective surgery. Surgery 128: 757-760. http://dx.doi.org/10.1067/msy.2000.107166

Qiao ZH and Tang LF (2010). The research progress of resistin and inflammatory diseases. Medical Recapitulate 16: 16321634.

Reilly MP, Lehrke M, Wolfe ML, Rohatgi A, et al. (2005). Resistin is an inflammatory marker of atherosclerosis in humans. Circulation 111: 932-939.http://dx.doi.org/10.1161/01.CIR.0000155620.10387.43

Steppan CM, Bailey ST, Bhat S, Brown EJ, et al. (2001). The hormone resistin links obesity to diabetes. Nature 409: $307-312$. http://dx.doi.org/10.1038/35053000

Tsatsoulis A, Mantzaris MD, Bellou S and Andrikoula M (2013). Insulin resistance: an adaptive mechanism becomes maladaptive in the current environment - an evolutionary perspective. Metabolism 62: 622-633. http://dx.doi.org/10.1016/j.metabol.2012.11.004

Wiesner G, Brown RE, Robertson GS, Imran SA, et al. (2006). Increased expression of the adipokine genes resistin and fasting-induced adipose factor in hypoxic/ischaemic mouse brain. Neuroreport 17: 1195-1198. http://dx.doi.org/10.1097/01.wnr.0000224776.12647.ba

Zhou Q, Yang J, Luo NS, Zhou Y, et al. (2011). Investigation of serum adiponectin, resistin and insulin resistance levels in the obese people. Jianyan Yixue 1: 23-26. 